\title{
Nanoscale Imaging and Control of Resistance Switching in Vo[sub]2 at Room Temperature
}

\section{Citation}

Kim, Jeehoon, Changhyun Ko, Alex Frenzel, Shriram Ramanathan, and Jennifer E. Hoffman. 2010. Nanoscale imaging and control of resistance switching in VO[sub]2 at room temperature. Applied Physics Letters 96 (213106).

\section{Published Version}

doi:10.1063/1.3435466

\section{Permanent link}

http://nrs.harvard.edu/urn-3:HUL.InstRepos:4342532

\section{Terms of Use}

This article was downloaded from Harvard University's DASH repository, and is made available under the terms and conditions applicable to Open Access Policy Articles, as set forth at http:// nrs.harvard.edu/urn-3:HUL.InstRepos:dash.current.terms-of-use\#OAP

\section{Share Your Story}

The Harvard community has made this article openly available.

Please share how this access benefits you. Submit a story.

\section{Accessibility}




\title{
Nanoscale Imaging and Control of Resistance Switching in $\mathrm{VO}_{2}$ at Room Temperature
}

\author{
Jeehoon Kim, ${ }^{1}$ Changhyun Ko, ${ }^{2}$ Alex Frenzel, ${ }^{1}$ Shriram Ramanathan, ${ }^{2}$ and Jennifer E. Hoffman ${ }^{1,}$ a) \\ ${ }^{1)}$ Department of Physics, Harvard University, Cambridge, MA 02138, U. S. A. \\ 2) School of Engineering and Applied Sciences, Harvard University, Cambridge, MA 02138, \\ U. S. A.
}

(Dated: 25 May 2010)

\begin{abstract}
We demonstrate controlled local phase switching of a $\mathrm{VO}_{2}$ film using a biased conducting atomic force microscope tip. After application of an initial, higher 'training' voltage, the resistance transition is hysteretic with $I V$ loops converging upon repeated voltage sweep. The threshold $V_{\text {set }}$ to initiate the insulator-to-metal transition is on order $\sim 5 \mathrm{~V}$ at room temperature, and increases at low temperature. We image large variations in $V_{\text {set }}$ from grain to grain. Our imaging technique opens up the possibility for an understanding of the microscopic mechanism of phase transition in $\mathrm{VO}_{2}$ as well as its potential relevance to solid state devices.
\end{abstract}

An insulator-to-metal transition may be triggered in $\mathrm{VO}_{2}$ as a function of temperature ${ }^{1}$, strain $^{2}$, electric field $^{3}$, or optical excitation ${ }^{4}$. This transition has useful properties such as fast 80 fs switching time ${ }^{5}$, high resistivity ratio, large change in optical reflectance ${ }^{6}$, and tunability near room temperature. Proposed applications include bolometers ${ }^{7}$, memristors ${ }^{8}$, tunablefrequency metamaterials ${ }^{9}$, and data storage ${ }^{10}$.

Sensor applications typically require negligible hysteresis, while memory applications call for maximum hysteresis, but all applications seek to maximize the resistivity ratio (RR). Single crystal $\mathrm{VO}_{2}$ exhibits $\mathrm{RR}$ up to $10^{5}$, but bulk single crystals pose problems for real devices due to cracking on repeated cycle through the transition ${ }^{11}$. Epitaxial films on insulating $\mathrm{Al}_{2} \mathrm{O}_{3}$ may have $\mathrm{RR}$ up to $10^{412}$. As a route to interface with existing electronics, recent effort has been devoted to film growth on $\mathrm{Si}$ substrates ${ }^{13}$, where voltage-controlled switching of $\mathrm{VO}_{2}$ in capacitor geometry has been demonstrated at room temperature $^{14}$. However, the lattice mismatch results in polycrystalline $\mathrm{VO}_{2}$ films with $\mathrm{RR}$ so far limited to $\sim 10^{3}$. It is therefore important to understand the effects of grain size and the role of grain boundaries in determining hysteresis loop properties RR, $V_{\text {set }}$, and $V_{\text {reset }}$. To date, the voltage triggered transition has been demonstrated down to $200 \mathrm{~nm}$, but only in a fixed area composed of multiple grains ${ }^{15}$. Here we present nanoscale images of the voltage-triggered transition: we resolve single grains as small as tens of nanometers.

We study a $200 \mathrm{~nm}$ thick $\mathrm{VO}_{2}$ film grown by rf sputtering from a $\mathrm{VO}_{2}$ target onto a heavily As-doped $\mathrm{Si}$ substrate $(n \text {-type, with resistivity } 0.002-0.005 \Omega \mathrm{cm})^{14}$. $\mathrm{X}$-ray diffraction data in Fig. 1a corresponds to a polycrystalline, monoclinic $\mathrm{VO}_{2}$ phase. Fig. $1 \mathrm{~b}$ shows the thermal phase transition with $\mathrm{RR}>10^{2}$. From AFM topography, the typical lateral diameter of a $\mathrm{VO}_{2}$ grain is $\sim 100 \mathrm{~nm}$, and the RMS surface roughness is $\sim 6 \mathrm{~nm}$.

To investigate this film, we use a home-built force microscope with a conducting cantilever of spring constant

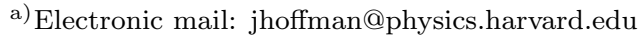

$k_{c}=40 \mathrm{~N} / \mathrm{m}^{17}$. We touch down on the surface with feedback to fix cantilever deflection at a typical setpoint of $\sim 4 \mathrm{~nm}$, corresponding to a force of $160 \mathrm{nN}$, and a pressure of $\sim 800$ bar (assuming a tip contact area diameter $\sim 50 \mathrm{~nm}$ ). The transition temperature has been shown to shift most significantly with $c$-axis uniaxial stress, at a rate of $-1.2 \mathrm{~K} / \mathrm{kbar}^{18}$. The force applied by the tip therefore corresponds to change in local transition temperature $\Delta T_{c}$ of at most $1 \mathrm{~K}^{19}$.

Upon first upward voltage sweep at a given location, we typically observe a sudden transition from the insulating to the metallic state at a 'training' voltage $V_{\mathrm{T}} \sim 12 \mathrm{~V}$. The transition is hysteretic, returning to the insulating state only at a much lower voltage $V_{\text {reset }}$. Subsequent sweeps show transitions at lower $V_{\text {set }}$, but the hysteresis remains, and $I V$ loops roughly stabilize with typical $\sim 5 \%$ jitter around $V_{\text {set }} \sim 5 \mathrm{~V}$ and $V_{\text {reset }} \sim 3 \mathrm{~V}$. This is comparable to the $3 \%$ stability of transition temperatures
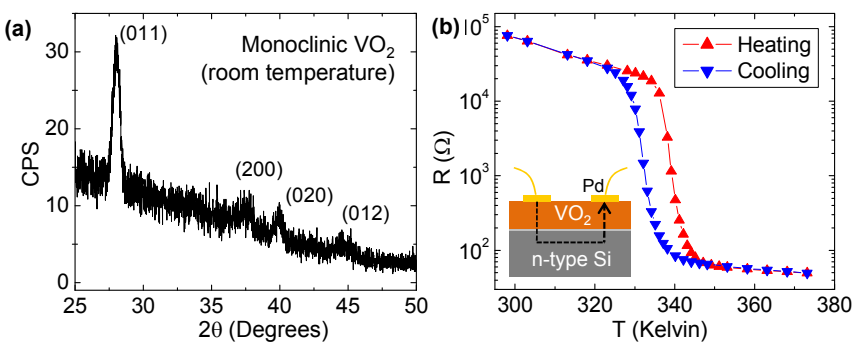

FIG. 1. (Color online) (a) X-ray diffraction (XRD) profile, performed on a Scintag XDS2000 diffractometer using $\mathrm{Cu} K \alpha$ radiation $(\lambda \sim 1.5418 \AA)$ at incidence angle of $1^{\circ}$. The observed peaks correspond to the monoclinic $\mathrm{VO}_{2}$ phase and demonstrate polycrystallinity. ${ }^{16}$ (b) The resistivity ratio is $>10^{2}$ as a function of temperature, measured in twopoint geometry as shown in the inset. For this data, voltage was applied and current measured between two neighboring $500 \times 500 \mu \mathrm{m}^{2} \mathrm{Pd}$ contact pads, centered $1 \mathrm{~mm}$ apart. Varying the distance between $\mathrm{Pd}$ pads did not alter the resistance, demonstrating that the resistance is entirely vertical through the $\mathrm{VO}_{2}$ film, with negligible contribution from the doped $\mathrm{Si}$ substrate or the $\mathrm{SiO}_{x}$ interface. 

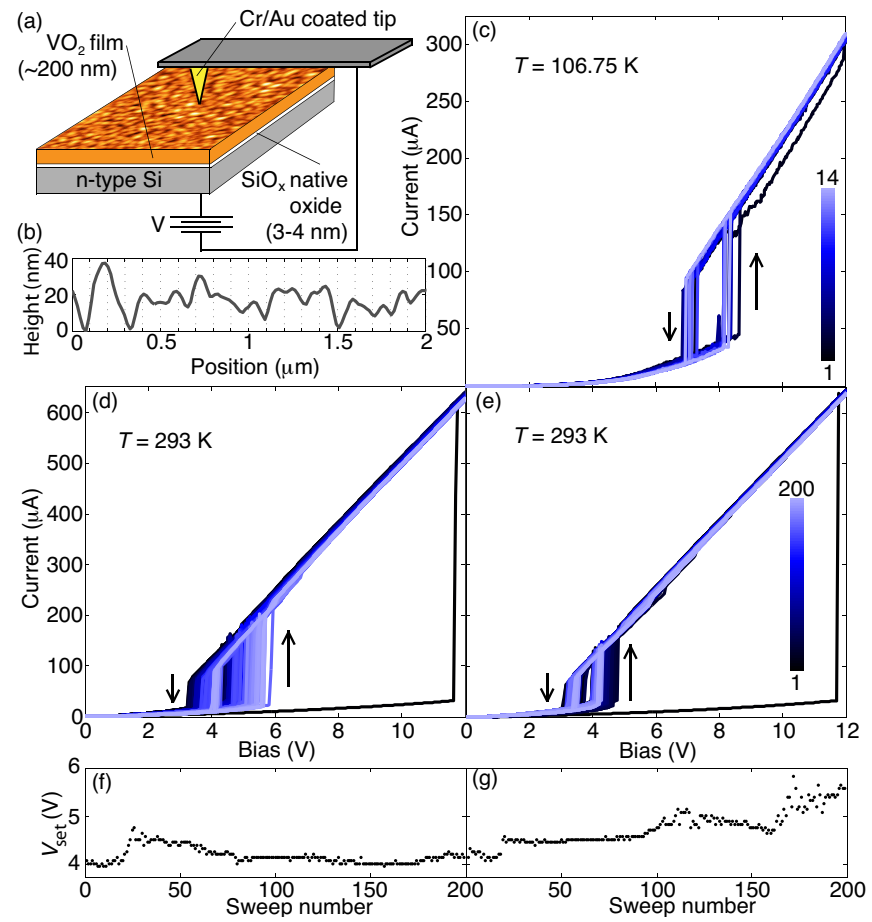

FIG. 2. (Color online) (a) Schematic of the microscope tip and sample geometry. (b) Height trace from AFM topography demonstrates the tip resolution and $\mathrm{VO}_{2}$ surface roughness. (c) 14 consecutive $I V$ sweeps at a single location at $T=107 \mathrm{~K}$. (d-e) 200 consecutive $I V$ sweeps at 2 different representative locations, at room temperature. Iterations start from black and run through light blue. Training voltage is $V_{\mathrm{T}} \sim 12 \mathrm{~V}$ in d-e and $>40 \mathrm{~V}$ in c. (f-g) $V_{\text {set }}$ as a function of iteration number for the data from d-e.

over 102 thermal cycles previously observed in a macroscopic junction on a $\mathrm{VO}_{2}$ film on $\mathrm{Al}_{2} \mathrm{O}_{3}$ substrate ${ }^{20}$. Typical voltage sweeps are shown in Fig. 2. The following points are worth noting in this unique measurement geometry: (1) Unlike in prior work on $200 \mathrm{~nm} \mathrm{Au} \operatorname{dots}^{15}$, we rarely see multiple jumps in a single curve; we really can access single grains! (2) As in this prior work, the measured $\mathrm{RR}$ for the entire tip- $\mathrm{VO}_{2}$ - $\mathrm{Si}$ structure is limited to $<10$, which we attribute to resistance $R_{s} \sim 15 \mathrm{k} \Omega$, in series with the $\mathrm{VO}_{2}$ film. (3) Loops do not depend on sweep speed from $6 \mathrm{~V} / \mathrm{s}$ to $16 \mathrm{~V} / \mathrm{s}$. (4) Loop characteristics exhibit negligible dependence on force within the range 120 to $420 \mathrm{nN}$ used in this study.

We next investigate the spatial dependence of the transition. After 'training' an area by scanning with a bias voltage of $11.1 \mathrm{~V}$, we rescan with increasing bias to watch the details of metallic puddle growth. As shown in Fig. 3, the insulating state displays variations in conductivity up to $100 \%$ of the mode value. Conductivity appears constant within each grain, but slowly varies from one grain to the next. Conductivity appears lower in the grain boundaries, which suggests a different stoichiometric phase in the grain boundaries. (We cannot rule out a topographic artifact from variations in the contact area of the tip between grains and grain boundaries, but such an effect would likely result in increased contact area in the grain boundaries, thus apparent higher conductivity.) On increasing voltage, the metallic state nucleates at the grains with largest insulating-state conductivity, grows into a larger metallic puddle, and shrinks again as the voltage is decreased. This type of switching is typically referred to as 'threshold switching' (as opposed to 'memory switching' in which the resistance state remains changed after removal of the applied voltage). Granularity and lower conductivity grain boundaries remain apparent in the metallic as well as the insulating phase.

Although previous researchers have suggested that field or carrier injection alone may be sufficient to induce the transition ${ }^{21,22}$, evidence suggests that in our experimental geometry the transition results most directly from Joule heating. At room temperature, the local power injection immediately prior to the insulatorto-metal transition shown in Fig. 2d, is $P \sim 100 \mu \mathrm{W}$. Given the specific heat $C=690 \mathrm{~J} /(\mathrm{K} \mathrm{kg})$ and the mass density $\rho=4340 \mathrm{~kg} / \mathrm{m}^{3}$ of $\mathrm{VO}_{2}$, the time to heat a

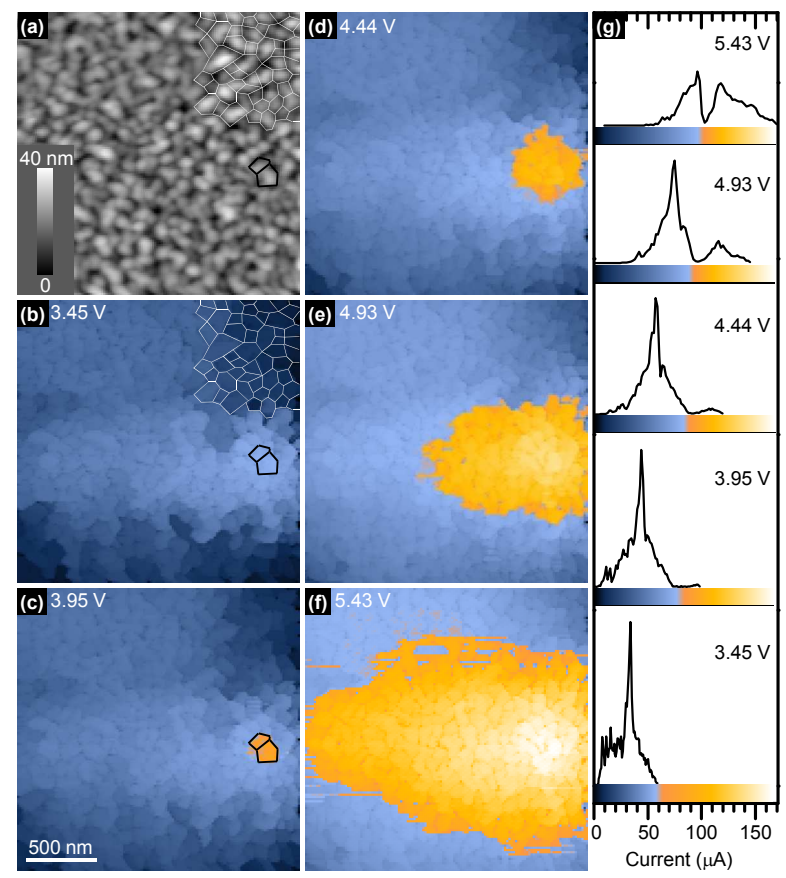

FIG. 3. (Color online) (a) AFM topography of the $\mathrm{VO}_{2}$ surface. (b-f) Current maps at increasing bias voltage show the metallic puddle seeded at 2 grains (outlined in black), growing with increasing bias. Grain boundaries are drawn in white in the upper right corners of a-b, which were acquired simultaneously, to emphasize the correlation between grain locations and regions of constant current. (g) Current distributions for b-f are bimodal, showing the jump between insulating and metallic state conductivity. A second set of images (not shown), acquired in this same area, as the applied voltage was subsequently decreased by the same increments, show the shrinking and disappearance of the metallic puddle. 
single grain of volume $V \sim(100 \mathrm{~nm})^{3}$ from room temperature $\left(T_{\mathrm{RT}} \sim 293 \mathrm{~K}\right)$ to the transition temperature $\left(T_{c} \sim 340 \mathrm{~K}\right)$ would be only $1.4 \mathrm{~ns}$. The empirical fact that the grain does not transition sooner implies significant thermal conduction away from each grain. The hysteresis may be explained as follows: on upwards voltage sweep, the switching occurs just as the Joule heating exceeds the heat flow out by enough to raise the grain temperature above its $T_{c}$. Upon transition, the current flow and resultant Joule heating suddenly increase, so the grain temperature does not immediately drop below $T_{c}$ as the voltage is swept back down. But the increased Joule heating at the higher current state causes the surrounding grains and Si substrate to also increase in temperature, which increases their thermal conductivity, so heat flows away more quickly. The grain may then return to the insulating state on downwards voltage sweep even though the power input is still higher than the power input on insulator-to-metal transition.

The hypothesis of Joule heating is further suggested by the following observations: (1) Transition occurs at the same absolute value of voltage, to within $\pm 10 \%$, for positive and negative applied bias. (2) The transition is seeded at the grains with the largest insulating state conductance (i.e. the largest Joule heating for a given applied voltage). (3) The transition can also be triggered by voltage at the much reduced global temperature of $107 \mathrm{~K}$, as shown in Fig. 2c, but the transition is shifted to higher voltage and power, as would be required to achieve the much larger $\Delta T=234 \mathrm{~K}$.

The origin of the training voltage remains unknown. We rule out surface contamination, because use of the tip to scrape the $\mathrm{VO}_{2}$ surface, in situ in vacuum, with enough force to physically remove up to $10 \%$ of the $\mathrm{VO}_{2}$ material, does not lower the initial transition voltage. Possibly, the training alters the native $\mathrm{SiO}_{x}$ layer. More likely, the training may occur within the $\mathrm{VO}_{2}$ film itself, possibly due to $\mathrm{O}$ migration which improves grain stoichiometry, or due to formation of more conductive phases such as Magneli $\left(\mathrm{V}_{n} \mathrm{O}_{2 n-1}\right)$ phases ${ }^{23}$ in the grain boundaries.

In conclusion, single grain switching with reproducible hysteresis demonstrates the scaling of the insulator-tometal transition in $\mathrm{VO}_{2}$ down to tens of nanometers. Nanoscale voltage-triggered switching at room temperature may lead to additional $\mathrm{VO}_{2}$ applications. High resolution conductance imaging of $\mathrm{VO}_{2}$ has the potential to elucidate the microscopic mechanism of phase transition, and to inform the optimization of grain size and grain boundary composition in practical $\mathrm{VO}_{2}$ film devices.

This work is supported by the Harvard NSEC under NSF Grant No. PHY-0117795, and by AFOSR Grant No. FA9550-08-1-0203.

${ }^{1}$ F. J. Morin, Physical Review Letters 3, 34 (1959).

${ }^{2}$ J. Cao, E. Ertekin, V. Srinivasan, W. Fan, S. Huang, H. Zheng, J. W. L. Yim, D. R. Khanal, D. F. Ogletree, J. C. Grossman, and J. Wu, Nature Nanotechnology 4, 732 (2009).

${ }^{3}$ G. Stefanovich, A. Pergament, and D. Stefanovich, J. Phys.: Condens. Matter 12, 8837 (2000).

${ }^{4}$ A. Cavalleri, C. Tóth, C. Siders, J. A. Squier, F. Ráksi, P. Forget, and J. C. Kieffer, Physical Review Letters 87, 237401 (2001).

${ }^{5}$ A. Cavalleri, T. Dekorsy, H. H. W. Chong, J. C. Kieffer, and R. W. Schoenlein, Physical Review B 70, 161102 (2004).

${ }^{6}$ D. Ruzmetov, K. T. Zawilski, S. D. Senanayake, V. Narayanamurti, and S. Ramanathan, Journal of Physics: Condensed Matter 20, 465204 (2008).

${ }^{7}$ C. Chen and Z. Zhou, Applied Physics Letters 91, 011107 (2007).

${ }^{8}$ T. Driscoll, H.-T. Kim, B.-G. Chae, M. D. Ventra, and D. N. Basov, Applied Physics Letters 95, 043503 (2009).

${ }^{9}$ T. Driscoll, S. Palit, M. M. Qazilbash, M. Brehm, F. Keilmann, B.-G. Chae, S.-J. Yun, H.-T. Kim, S. Y. Cho, N. M. Jokerst, D. R. Smith, and D. N. Basov, Applied Physics Letters 93, 024101 (2008).

${ }^{10}$ M.-J. Lee, Y. Park, D.-S. Suh, E.-H. Lee, S. Seo, D.-C. Kim, R. Jung, B.-S. Kang, S.-E. Ahn, C. B. Lee, D. H. Seo, Y.-K. Cha, I.-K. Yoo, J.-S. Kim, and B. H. Park, Advanced Materials 19, 3919 (2007).

${ }^{11}$ J. A. S. Barker, H. W. Verleur, and H. J. Guggenheim, Phys. Rev. Lett. 17, 1286 (1966).

${ }^{12}$ M. M. Qazilbash, M. Brehm, B.-G. Chae, P.-C. Ho, G. O. Andreev, B.-J. Kim, S. J. Yun, A. V. Balatsky, M. B. Maple, F. Keilmann, H.-T. Kim, and D. N. Basov, Science 318, 1750 (2007).

${ }^{13}$ D. Ruzmetov, K. T. Zawilski, V. Narayanamurti, and S. Ramanathan, Journal of Applied Physics 102, 113715 (2007).

${ }^{14}$ C. Ko and S. Ramanathan, Journal of Applied Physics 106, 034101 (2009).

${ }^{15}$ D. Ruzmetov, G. Gopalakrishnan, J. Deng, V. Narayanamurti, and S. Ramanathan, Journal of Applied Physics 106, 083702 (2009).

16 JCPDS Card No. 01-082-0661.

${ }^{17} \mathrm{http}: / /$ www.spmtips.com/nsc/16/cr-au.

${ }^{18}$ L. A. Ladd and W. Paul, Solid State Communications 7, 425 (1969).

${ }^{19}$ Our attempts to use smaller forces with $k_{c}=0.15 \mathrm{~N} / \mathrm{m}$ and $k_{c}=$ $3 \mathrm{~N} / \mathrm{m}$ spring constant cantilevers did not result in reproducible resistance measurements.

${ }^{20} \mathrm{C}$. Ko and S. Ramanathan, Journal of Applied Physics 104, 086105 (2008).

${ }^{21}$ G. Gopalakrishnan, D. Ruzmetov, and S. Ramanathan, J. Mater. Sci. 44, 5345 (2009).

${ }^{22}$ M. M. Qazilbash, Z. Q. Li, V. Podzorov, M. Brehm, F. Keilmann, B. G. Chae, H. T. Kim, and D. N. Basov, Applied Physics Letters 92, 241906 (2008).

${ }^{23}$ S. Kachi, K. Kosuge, and H. Okinaka, J. Solid State Chem. 6, 258 (1973). 